\title{
PHYTOSOCIOLOGICAL STUDIES ON THE STEPPE COMMUNITIES OF NATURAL PASTURES IN ŞANLIURFA, UPPER MESOPOTAMIA, TURKEY
}

\author{
CEVHERI, C. $.^{*}-$ ASLAN, M. $^{2}-$ AtAMOV, V. ${ }^{3}$ \\ ${ }^{1}$ Department of Biology, Faculty of Science and Art, Harran University, Sanluurfa, Turkey \\ ${ }^{2}$ Faculty of Education, Harran University, Şanliurfa, Turkey \\ ${ }^{3}$ Department of Biology, Faculty of Science and Art, Recep Tayyip Erdoğan University, Rize, \\ Turkey \\ *Corresponding author \\ e-mail: ccevheri@harran.edu.tr; phone: +90-414-318-3566
}

(Received 28 $8^{\text {th }}$ May 2019; accepted $28^{\text {th }}$ Nov 2019)

\begin{abstract}
This phytosociological study was carried out in the steppe of Fatik Mountains in Sanliurfa, between the years 2002-2005. At the end of this study, it was underlined that the dominant vegetation type was steppe in the region and different steppe associations were determined. These were also; Festuco callieri-Teucrietum poliae ass. nova, Astragalo strictifolii-Salvietum sclarea ass. nova, Phlomido bruguieri-Thymbretum spicatae ass. nova, Asphodelo aestivum-Teucrietum poliae ass. nova, Cynodo dactyloni-Bromoetum danthoniae ass. nova, and Prosopo farctae-Avenetum erianthae ass. nova. During the floristic surveys, 153 taxa belonging to 26 families and 107 genera were identified. Poaceae with 31 taxa (20.3\%), Asteraceae with 24 taxa (15.7\%), Fabaceae with 22 (14.4\%), and Apiaceae with 13 taxa $(8.5 \%)$ were the richest families.
\end{abstract}

Keywords: steppe vegetation, grazing, ecosystems, characteristic species, association

\section{Introduction}

Turkey is located at the intersection of two important gene centers namely the Mediterranean and Near East and has the ninth highest biodiversity among terrestrial countries. Natural species of most cereals cultivated for human consumption grow on steppe ecosystems (Ture and Bocuk, 2007).

Fatik Mountain, located at the southwestern part of Şanliurfa, was selected as the research area since it has a peculiar steppe vegetation and has been paid less attention so far as "Adıgüzel and Aytac, 2001" mentioned earlier. The studied area is in the C7 square according to grid system adopted by Davis (1988). Phytogeographically located in the Irano-Turanian Floristic Region, extending between the longitudes of 37.2 North and 37.3 and between the latitudes of 38.6 and 38.7 East (Anonymous, 1995).

The central, eastern and southeastern parts of Turkey belong phytogeographically to the Irano-Turanian region (Zohary, 1973). The Irano-Turanian region is separated into two parts the West and the East Irano-Turanian subregions. There are three provinces in Turkey belonging to the West Irano-Turanian subregion namely; Central Anatolian, Irano-Anatolian and the Mesopotamian provinces. According to this classification, Southeast Anatolia is located in the Mesopotamian province of the West Irano-Turanian subregion (Zohary, 1973).

According to Flora of Davis (Davis 1965-1985; Davis et al.; 1988; Güner et al., 2000) some researchers among them Kotschy, Sintenis, Davis, and Zohary have visited and 
gathered plant specimens from Şanluurfa by Baytop (2003). Some floristic and taxonomic studies were carried out in and around Southeast Anatolia (Kaynak and Ketenoğlu, 1980; Malyer, 1981-1983; Kaynak, 1987-1989a, b; Y1ldırımlı, 1992-1994; Yıldız and Aktoklu, 1996; Ertekin and Saya, 1997; Ertekin, 2002; Adigüzel and Aytac, 2001; Aslan and Turkmen, 2001-2003; Turkmen et al., 2002; Akan et al., 2005; Aydoğdu and Akan, 2005). But there has been no vegetational research data except for a study carried out by Zohary (1973). In addition, Southeast Anatolia is one of the most little known or unknown regions of Turkey (Davis, 1965-1985; Çırpıcı, 1987; Donner, 1990).

In the region, the natural areas are used as pastures like in all arid countries. Artificial pasture culture has not been demanded yet. It has been stated that it contains wheat, beans in pastures in arid regions and includes drought-resistant different vegetation types and other herbaceous plants such as Savanne, Praerie, Steppe, Maguis, Phyrgana. (Gençkan, 1983). This understanding and using of pastures are applied in Şanliurfa on large scale, like in the most of regions of Turkey. In this region the open places which are out of using, belong to state, like slopes, stony, rocky places, stream beds, hills etc. (Çullu et al., 2002). Şanlıurfa is one of little researched region in Turkey (Aslan, 2015). It has 724.529 ha of area. 263.507 ha of this area is used as natural pastures. In the region, the pastures have the poorest plant cover (10-15\%) in Turkey. 0.82 ha of area is divided for each cow. In the past years there have been significant changes in the diversity of plants in this area due to random overgrazing and in some years fires (steppe fires) due to the abundance of animals. But, when the pastures of the region are taken into consideration it is necessary to divide 4 ha area for each cow. This shows that the pastures are used five times excessively. Excessive and irregular using have caused succession and degradation. The primary vegetation has transformed to secondary vegetation and converting towards semi-desert ecosytem (Atamov et al., 2004).

The characteristic vegetation type is steppe in this region (Atamov et al., 2004). The threat factors are excessive grazing and transforming of natural pastures to agricultural areas. Because of increased industrial activities, urbanization, tourism activities, creating new agricultural areas, mining activities, using agricultural methods and overgrazing, the natural structures of steppe ecosystems have been getting destroyed (Ture and Bocuk, 2007). 74 taxa are under threat and of these 10 taxa on Karacadağ, 13 taxa in Ceylanpınar are endemic (Özhatay et al., 2003; Aslan, 2018). In Şanlıurfa, the steppe vegetation is dominant because of ecological, geographical and antropogenic factors. Usually, animals eat legumes, grasses and similar crops, which are fresh and delicious for a period of one year. However, the perennial shrub, smelly and prickly plants are not preferred.

Under effects of topographic and edafic factors, different steppe vegetation types are found in the region. In this steppe, wheats are dominant and different plants can be found, which have life forms like geophytes, camephytes, cryptophytes, hemicryptohytes and terophytes. In some districts, some remains of forest and rare old trees are found. In addition, the efemeres which grow rapidly and complete their life cycles in short time after spring and autumn rains are present in the vegetation.

The constant plants are perennial herbs, shrubs and trees that are seen rarely (Atamov et al., 2007). But, the efemeres are found and affect quality of fodder plants and yields positively.

In the region, watery agriculture has been made for years. Agricultural method applied to irrigation in semi-arid and arid regions depending on the characteristics of the plant sown or to obtain higher yields. Since these areas are not natural areas, weed yield 
is considerably reduced due to the excess number of animals. Holophytic areas have risen $394 \%$. The agriculture of wheat and cotton have been stopped in some places because of salinity and these areas have been used as pastures for the last 15 years (Çullu et al., 2002).

\section{Materials and methods}

The city Şanlıurfa in Southeast of Anatolia is between $37^{\circ} 49^{\prime} 12^{\prime \prime}-40^{\circ} 10^{\prime}$ '00" East meridian and $36^{\circ} 41^{\prime} 28^{\prime \prime}-37^{\circ} 55^{\prime}$ '50" North parallel. In Şanliurfa the uneven areas are at percentage of $60.4 \%$, mountains $22 \%$, plains $16.3 \%$ and plateaus $1.3 \%$. Harran plain, covering the study area, is one of the most important agricultural areas of Turkey and located between $36^{\circ} 47^{\prime}$ and $39^{\circ} 15^{\prime}$ east longitude and $36^{\circ} 40^{\prime}$ and $37^{\circ} 41^{\prime}$ north latitude within the borders of the province of Şanlıurfa in Southeastern Anatolian region. The most important plains are named Harran, Suruç, Viranşehir, stayed on south part of Şanlıurfa. Ceylanpınar plain are stayed on southwest. In addition, Halfeti, Hilvan and Bozova plains have large areas. The average altitude is $375 \mathrm{~m}$. The lowest plain is Harran plain. Viranşehir plain is at east, Suruç plain is at west of Harran plain. It is 141 535 ha (Anonymous, 1995) (Figs. 1, 2 and 3).

Floristic and phytosociologic research studies were carried out between the years 2000-2005 at Karacadağ, Tektek, Fatik, Kaşmer, Nemrut, Kalecik mountains and Direkli Hills where are used as natural pastures. Regular geobotanical trips were organized. Plant specimens were collected and preserved according to the herbarium techniques. These were identified with the help of "Flora of Turkey and the East Aegean Islands" (Davis, 1965-1985, 1988; Güner et al., 2000) and other relevant publications (Akman et al., 2001) as well as the flora of Syria, Iraq and Iran. In addition, the plants of Birecik Dam lake area and halophytic plants in Akçakale were gathered and classified (Ertekin and Saya, 1997; Ertekin, 2002; Adigüzel and Aytac, 2001; Aslan, 2002; Aslan and Türkmen, 2001-2003; Kaya, 2002; Atamov et al., 2004; Akan et al., 2005; Aydın, 2003; Aydoğdu and Akan, 2005; Ayalp, 2005; Atamov et al., 2005).

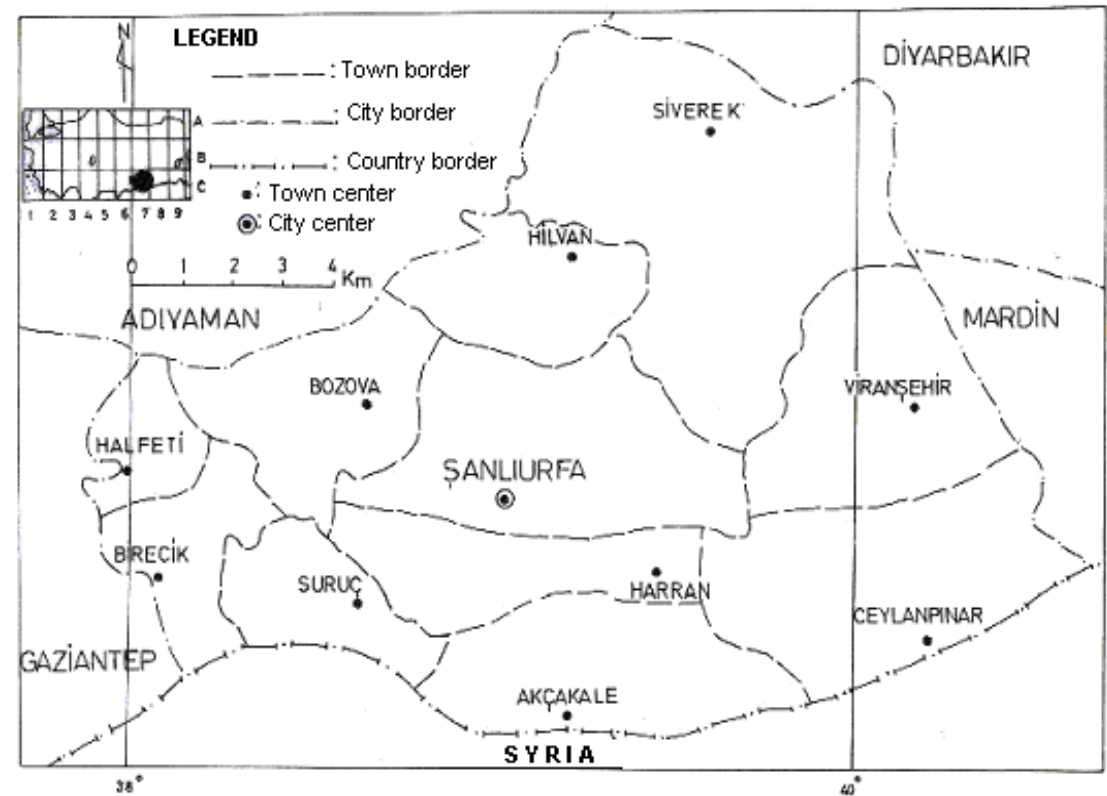

Figure 1. Location of the study area in Turkey 


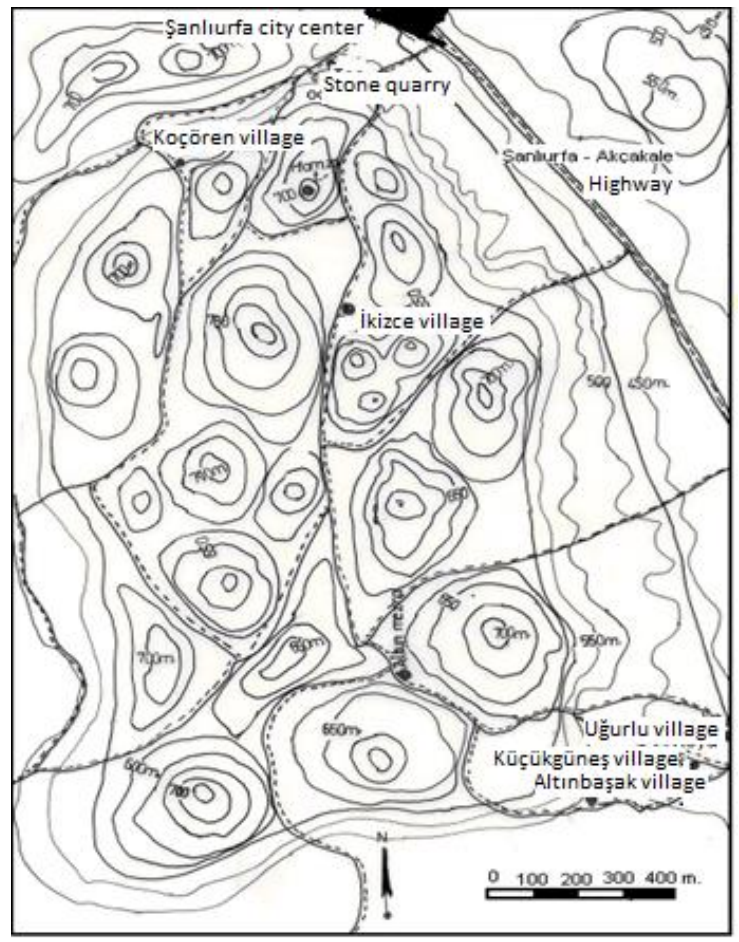

Figure 2. The map of Fatik Mountains

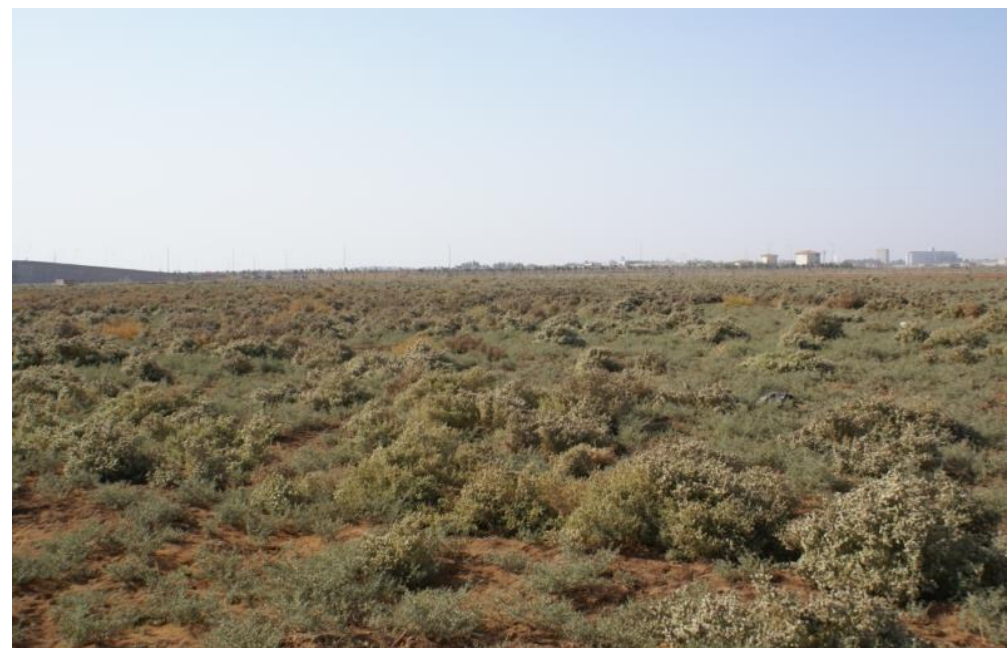

Figure 3. A photo of the research area

Braun-Blanquet's (1964) method was used for the naming of phytosociological plant groups. Plants were sampled twice a month during certain vegetation periods and plant samples were taken to identify the plants and examine the necessary materials.) These plants have been protected in the Herbarium of Harran University. The climatic data were taken from the Sanliurfa Meteorology Station and has been evaluated according to these data. Approximately $500 \mathrm{~g}$. soil samples were taken from $5 \mathrm{~cm}$ under the soil in the research areas in spring, summer and autumn. The soil samples were dried naturally and analysis of them were made by the Ministry of Agriculture and the Rural Affairs General Directorate of Rural Services Sanliurfa Research Institute. 


\section{Results and discussion}

As it is seen in Figure 4 and Table 1, the climate in Şanliurfa is arid from June to October in a long period. In this period, most annual plants die. Only perennial herbs, shrubs and trees are alive. Semiarid Mediterranean climate occurs in research area. According to Emberger the precipitation-temperature coefficient (Q) is 42.94 (Akman, 1990). Annual mean temperature is $18.7^{\circ} \mathrm{C}$. The maximum mean temperature (M) is $46.8{ }^{\circ} \mathrm{C}$, in July. The minimum mean temperature $(\mathrm{m})$ is $-6.8{ }^{\circ} \mathrm{C}$, in February. Annual rainfall is about $457.8 \mathrm{~mm}$ (Anonymous, 2001) and the seasonal precipitation regime is winter, spring, autumn and summer. This is the first variant of the East Mediterranean precipitation regime. The ombrothermic diagram shows dry and rainy periods (Fig. 4).

Table 1. The bioclimate and fall regime of Sanliurfa

\begin{tabular}{c|c|c|c|c|c|c|c|c}
\hline Altitude (m) & $\mathbf{P}(\mathbf{m m})$ & $\mathbf{M}\left({ }^{\circ} \mathbf{C}\right)$ & $\mathbf{M}\left({ }^{\circ} \mathbf{C}\right)$ & $\mathbf{Q}$ & $\mathbf{P E}$ & $\mathbf{S}$ & Fall regime & Bioclimate \\
\hline 547 & 457.8 & 46.8 & -6.8 & 42.94 & 7.2 & 0.18 & W.Sp.A.Su & Semi-arid, cold in winter \\
\hline
\end{tabular}

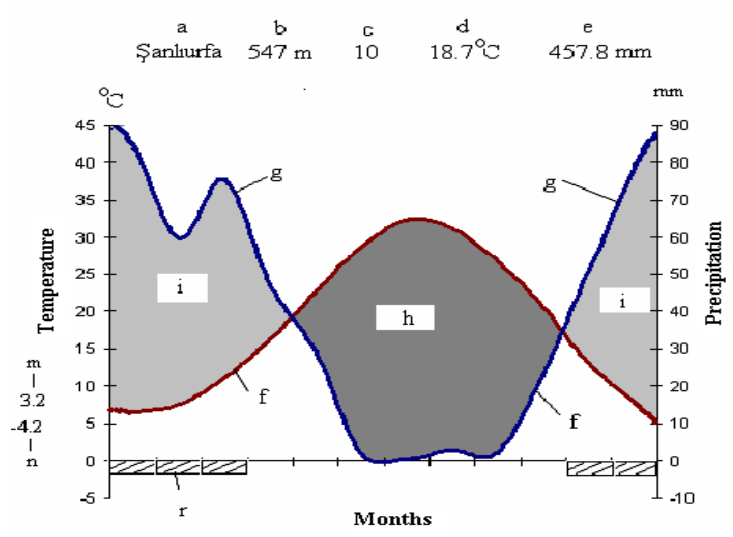

Figure 4. The climatic diagram of Shanluurfa. a: Meteorology station; $\boldsymbol{b}$ : The elevation of the Meteorology station $(m) ; \boldsymbol{c}$ : The year of temperature and precipitation; $\boldsymbol{d}$ : The mean annual temperature $\left({ }^{\circ} \mathrm{C}\right) ; \boldsymbol{e}:$ The mean annual precipitation $(\mathrm{mm}) ; \boldsymbol{f}:$ The curved line of temperature; $\mathrm{g}$ :

The curved line of precipitation; $\boldsymbol{h}:$ Arid season; $\boldsymbol{i}$ : Humid season; $\boldsymbol{m}:$ The least mean temperature of the most cold month $\left({ }^{\circ} \mathrm{C}\right) ; \boldsymbol{n}$ : Absolute minimum temperature $\left({ }^{\circ} \mathrm{C}\right) ; \boldsymbol{r}$ : Probable frosty month

In the different plant associations in the steppe vegetation, the avarage productivity changes between 1.0-7.0 $\mathrm{kg} \mathrm{m}^{-2}$. Excessive aridity and temperature have caused decreasing productivity and quality of fodder plants in pastures. From the end of autumn to the middle of spring, the efemeres grow and these plants increase the quality and productivity of fodders by 2-3 times. At the end, yield of natural pastures, number of fodder plant species and their populations have been reduced day to day because of excessive and unconscious grazing, migration, negative habitat conditions like aridity and temperature. The plants which have prickle, etheric oils, latex, poison, bitter and fluff have spread out. Excessive grazing has caused the transforming of the primary steppe to secondary and then semi-desert to desert vegetation.

In most of the places in this area, the covering degree of the flora is 40-50\%. And this is the characteristic property of half-desert and desert type associations. The flora 
has generally made up of a single layer and the average height of the grass covering is $10-20 \mathrm{~cm}$.

On the contrary, the number of plants which are not eaten by animals have been increased. Thus, the primary steppe vegetation has been transformed to secondary and it is seen that the succession has been transformed to semi-desert conditions.

Immigration is widespread in this region and emigrants migrate from East Anatolia to use pastures for grazing. Thousands of sheep destroy the all immature and senile groups of plants. The yield of pastures has been decreased. This situation has caused transforming from steppe to desertation.

In the flora of Şanlıurfa, 71 families, 798 taxa belonging to 342 genera have been determined (Atamov et al., 2004). 135 of these plants were monocotyledonaes, 663 were dicotyledonaes.

The number of plants belonging to Poaceae family were more than others in spite of having less species (56) than Fabaceae (101). The dominant taxa which spread out in naturel pastures were as follows: Bromus japonicus Thunb. subsp. japonicus, $B$. squarrosus L., B. sterilis L., B. tectorum L., Avena sterilis L. subsp. sterilis, Aegilops triuncialis L. subsp. triuncialis, A. neglecta Req. ex Bertol, Festuca callieri (Hackel ex St. - Yves) F. Markgraf subsp. callieri, Hordeum murinum L. subsp. glaucum (Steudel) Tzvelev.

Some plants belonging to Fabaceae, have fodder quality. Most of them are used as fodders. These were as follows; Astragalus onobrychis L., A. aleppicus Boiss., A. immarginatus Labm., A. dactylocarpus Boiss., Medicago rigidula (L.) var. rigidula, Onobrychis caput-galli (L.) Lam. O. crista-galli (L.) Lam. O. galegifolia Boiss. $O$. kotschyana Fenzl. Trifolium scabrum L., T. speciosum Willd., T. dasyurum C. Presl, T. angustifolium L. var intermedium, T. boisseri Guss. ex Boiss., T. meironense Zoh., T. campestre Schreb., Trigonella monspeliaca L., T. mesopotamica Hub. Mor., Vicia mollis Boiss., Coronilla orientalis Miller var. orientalis, C. scorpioides (L.) Koch, Hedysarum varium Willd.

In the research area, 153 taxa belonging to 26 families and 107 genera were identified. Poaceae with 31 taxa (20.3\%), Asteraceae with 24 taxa (15.7\%), Fabaceae with 22 (14.4\%), and Apiaceae with 13 taxa (8.5\%) were the richest families.

Braun-Blanquet's (1964) method was used for the naming of phytosociological plant groups.

32 sample parcels were evaluated and 6 new associations and their upper categories are as follows:

Astragalo-Bromotea Quezel 1973

Astragalo-Bromotalia Quezel 1973

Agropyro-Stachyon Quezel 1973

1. Festuco callieri-Teucrietum poliae ass. ova

Onobrychido armenae-Thymetalia leucostomi Akman, Ketenoglu Quezel 1984

2. Astragalo strictifolii-Salvietum sclarea ass. nova

3. Phlomido bruguieri-Thymbretum spicatae ass. nova

4. Asphodelo aestivum-Teucrietum poliae ass. nova

5. Cynodo dactyloni-Bromoetum danthoniae ass. nova

6. Prosopo farctae-Avenetum erianthae ass. nova 


\section{Festuco callieri-Teucrietum poliae ass. nova}

There were no trees and shrubs in its structure. Only grass layer was present and covering was $40-80 \%$. The length was between 10 and $35 \mathrm{~cm}$ (Table 2).

Festuco callieri-Teucrietum poliae association was spreading out where the calcereous main rock, gravelled and small pit areas. According to chemical and physical properties of soils where the association was spreading out homogenous, $\mathrm{pH}$ was between 7.0 and 7.71, the rate of lime $\left(\mathrm{CaCO}_{3}\right)$ was $3.8 \%$, phosphorus $\left(\mathrm{P}_{2} \mathrm{O}_{5}\right)$ was 5.2-5.3 kg dec $\mathrm{kg}^{-1}$; potassium $\left(\mathrm{K}_{2} \mathrm{O}\right)$ was $118.8-308.8 \mathrm{~kg} \mathrm{dec}^{-1}$ and organic component was $2.17-2.48 \%$ (Table 3).

Table 2. Festuco callieri-Teucrietum poliumae ass. nova. Thypus: Sample Parcel 62

\begin{tabular}{|c|c|c|c|c|c|c|c|c|c|c|c|}
\hline Sample parcel No & 12 & 23 & 34 & 35 & 37 & 41 & 42 & 46 & 48 & 50 & \\
\hline Square size $\left(\mathrm{m}^{2}\right)$ & 50 & 50 & 50 & 50 & 50 & 50 & 50 & 50 & 50 & 50 & \\
\hline Altitude (m) & 500 & 478 & 530 & 540 & 570 & 510 & 515 & 530 & 535 & 550 & \\
\hline Gradient (\%) & 20 & 30 & 30 & 35 & 30 & 25 & 30 & 35 & 30 & 25 & d \\
\hline Direction & $\mathrm{N}$ & NW & NW & $\mathrm{N}$ & $\mathrm{N}$ & $\mathrm{N}$ & $\mathrm{N}$ & $\mathrm{N}$ & $\mathrm{N}$ & NW & 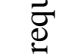 \\
\hline Length of cover $(\mathrm{cm})$ & 10 & 25 & 20 & 17 & 20 & 22 & 20 & 25 & 25 & 20 & \\
\hline Number of species & 21 & 21 & 20 & 16 & 17 & 18 & 18 & 15 & 16 & 17 & \\
\hline Cover $(\%)$ & 60 & 55 & 55 & 60 & 65 & 65 & 60 & 60 & 60 & 65 & \\
\hline \multicolumn{12}{|c|}{ Characteristic species of Association } \\
\hline Festuca callieri & 22 & 22 & +2 & +2 & +1 & 22 & 22 & 33 & 22 & 11 & $\mathrm{~V}$ \\
\hline Teucrium polium & - & 22 & +1 & 11 & 11 & 1 & 33 & 33 & +1 & 11 & IV \\
\hline Trifolium tomentosum & +2 & +2 & +1 & +2 & +2 & . & . & . & . & . & III \\
\hline
\end{tabular}

Characteristic species of Allians Festuca-Teucrion

\begin{tabular}{l|c|c|c|c|c|c|c|c|c|c|c}
\hline Phleum phleoides & +1 & +2 & +2 & +2 & $\cdot$ & $\cdot$ & $\cdot$ & +2 & +2 & +2 & III \\
Phlomis bruguieri & +2 & +2 & +2 & +2 & +2 & +2 & +2 & +1 & $\cdot$ & $\cdot$ & III \\
Eryngium creticum & +1 & +2 & +2 &. & $\cdot$ & +2 & +1 & $\cdot$ & $\cdot$ & $\cdot$ & II \\
\hline
\end{tabular}

Characteristic species of Ordo Daphno-Festucetalia

\begin{tabular}{l|l|l|l|l|l|l|l|l|l|l|l} 
Thymbra spicata var. spicata & 22 & 22 & 22 & 11 & 11 & +2 & +2 & +2 &. &. & III
\end{tabular}

Lolium rigidum

\begin{tabular}{ll|l|l|l|l|l|l|l|l|l|l} 
Hordeum murinum subsp. glaucum & +2 &. &. & +2 & +2 & +2 & +2 & +2 & +2 & . & III
\end{tabular}

Characteristic species of Classis Daphno-Festucetea

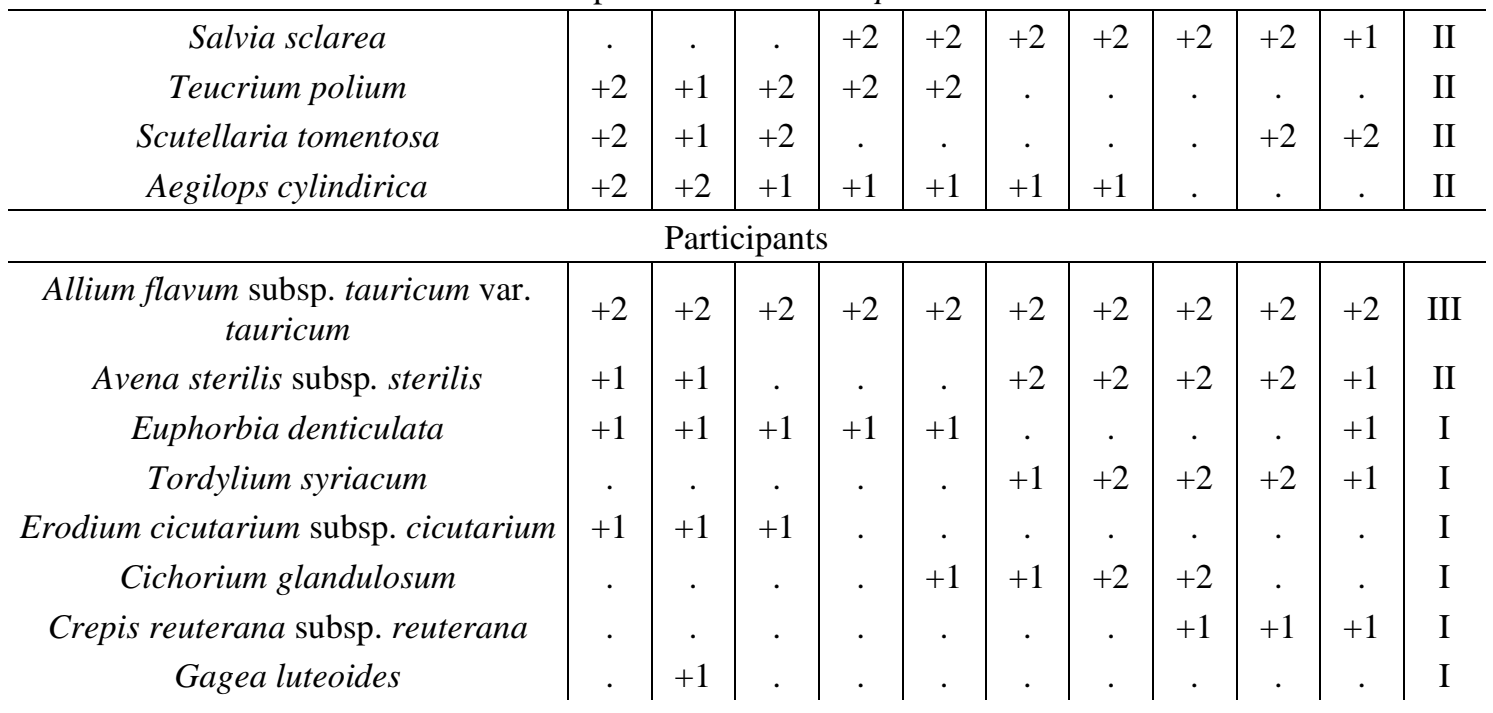




\begin{tabular}{l|c|c|c|c|c|c|c|c|c|c|c} 
Thlaspi perfoliatum & +1 & +1 & +1 & +1 & $\cdot$ & $\cdot$ & $\cdot$ & $\cdot$ & $\cdot$ & $\cdot$ & I \\
Muscari comosum & $\cdot$ & $\cdot$ & $\cdot$ & $\cdot$ & $\cdot$ & $\cdot$ & +1 & +1 & +1 & $\cdot$ & I \\
Scandix pecten-veneris & +1 & $\cdot$ & $\cdot$ & $\cdot$ & $\cdot$ & $\cdot$ & +1 & $\cdot$ & $\cdot$ & +1 & I \\
Lappula barbata & $\cdot$ & $\cdot$ & $\cdot$ & $\cdot$ & +1 & $\cdot$ & $\cdot$ & $\cdot$ & $\cdot$ & $\cdot$ & I \\
Cynosurus echinatus & $\cdot$ & +1 & $\cdot$ & $\cdot$ & $\cdot$ & $\cdot$ & $\cdot$ & $\cdot$ & $\cdot$ & $\cdot$ & I \\
Torilis tenella & +1 & +1 & $\cdot$ & $\cdot$ & $\cdot$ & $\cdot$ & $\cdot$ & $\cdot$ & +1 & $\cdot$ & I \\
Hypecoum imberbe & $\cdot$ & $\cdot$ & +1 & $\cdot$ & $\cdot$ & $\cdot$ & $\cdot$ & $\cdot$ & $\cdot$ & +1 & I \\
Senecio vulgaris & $\cdot$ & $\cdot$ & $\cdot$ & $\cdot$ & $\cdot$ & +1 & $\cdot$ & $\cdot$ & $\cdot$ & $\cdot$ & I \\
Anthemis haussknechtii & $\cdot$ & $\cdot$ & $\cdot$ & $\cdot$ & +1 & +1 & +1 & $\cdot$ & $\cdot$ & +1 & I \\
Gypsophila antari & +1 & +2 & +2 & +2 & $\cdot$ & $\cdot$ & $\cdot$ & +1 & $\cdot$ & $\cdot$ & II \\
Medicago minima var. minima & +1 & +1 & +2 & +2 & +2 & $\cdot$ & $\cdot$ & $\cdot$ & +2 & +2 & II \\
Echinops microcephalus & $\cdot$ & $\cdot$ & +1 & $\cdot$ & $\cdot$ & $\cdot$ & $\cdot$ & $\cdot$ & $\cdot$ & $\cdot$ & I \\
Centaurea solstitialis subsp. solstitialis & +1 & $\cdot$ & $\cdot$ & $\cdot$ & $\cdot$ & $\cdot$ & $\cdot$ & $\cdot$ & $\cdot$ & $\cdot$ & I \\
Hypecoum pendulum & $\cdot$ & $\cdot$ & $\cdot$ & $\cdot$ & $\cdot$ & $\cdot$ & $\cdot$ & $\cdot$ & +1 & +1 & I \\
Torilis leptophylla & $\cdot$ & $\cdot$ & +2 & +1 & +1 & +1 & +1 & $\cdot$ & $\cdot$ & $\cdot$ & I \\
\hline
\end{tabular}

Table 3. Chemical characteristics of the soil of the research area

\begin{tabular}{c|c|c|c|c|c|c|c|c}
\hline Location & $\begin{array}{c}\text { Deepth } \\
(\mathbf{c m})\end{array}$ & $\begin{array}{c}\text { Saturation } \\
\text { with water }(\boldsymbol{\%})\end{array}$ & $\begin{array}{c}\text { Total salt } \\
(\mathbf{\%})\end{array}$ & $\mathbf{p H}$ & $\begin{array}{c}\mathbf{C a C O}_{3} \\
(\mathbf{\%})\end{array}$ & $\mathbf{P}_{2} \mathbf{O}_{5}$ & $\mathbf{K}_{2} \mathbf{O}$ & $\begin{array}{c}\text { Organic } \\
\text { mass (\%) }\end{array}$ \\
\hline Ugurlu Village & $0-20$ & 77 & 0.071 & 7.70 & 3.8 & 5.3 & 118.8 & 2.48 \\
Kocoren Village & $0-20$ & 77 & 0.060 & 7.71 & 3.8 & 5.2 & 308.8 & 2.17 \\
Ikizce Village & $0-20$ & 68 & 0.073 & 7.28 & 2.6 & 4.9 & 179.2 & 1.44 \\
Altun Village & $0-20$ & 66 & 0.098 & 7.42 & 6.8 & 4.5 & 108 & 2.35 \\
\hline
\end{tabular}

The characteristic species of the association were Festuca callieri subsp. callieri, Teucrium polium. Each species are the elements of Irano-Turanian phytogeographic region.

Classis: Daphno-Festucetea Quezel 1964

Ordo: Daphno-Festucetalia Quezel1964

Alliance: Festuca-Teucrion ass. nova

\section{Asphodelo aestivi-Teucrietum poliae ass. nova}

The characteristic species of the association were Asphodelus aestivus and Teucrium polium. This association was at the north part of the Fatik Mountains and at the environs of Stone quarry. 10 sample areas were used. The gradient was $35-40 \%$, The length was between 600 and $700 \mathrm{~m}$ (Table 4).

Table 4. Asphodelo aestivi-Teucrietum poliae ass. nova. Thypus: Sample Parsel 63

\begin{tabular}{|c|c|c|c|c|c|c|c|c|c|c|c|}
\hline Sample parcel No & 60 & 61 & 62 & 67 & 70 & 73 & 77 & 79 & 81 & 82 & \\
\hline Square size $\left(\mathrm{m}^{2}\right)$ & 50 & 50 & 50 & 50 & 50 & 50 & 50 & 50 & 50 & 50 & \\
\hline Altitude (m) & 531 & 535 & 500 & 510 & 515 & 520 & 530 & 505 & 500 & 517 & \\
\hline Gradient (\%) & 30 & 20 & 25 & 25 & 30 & 30 & 35 & 30 & 35 & 35 & 这 \\
\hline Direction & $\mathrm{W}$ & $\mathrm{W}$ & $\mathrm{W}$ & $\mathrm{W}$ & NW & NW & NW & NW & NW & $\mathrm{W}$ & \\
\hline
\end{tabular}




\begin{tabular}{|c|c|c|c|c|c|c|c|c|c|c|c|}
\hline Length of cover $(\mathrm{cm})$ & 20 & 25 & 20 & 20 & 25 & 15 & 20 & 25 & 20 & 30 & \\
\hline Number of species & 14 & 13 & 11 & 8 & 12 & 13 & 13 & 10 & 11 & 11 & \\
\hline Cover $(\%)$ & 55 & 50 & 45 & 55 & 50 & 55 & 60 & 50 & 40 & 45 & \\
\hline \multicolumn{12}{|c|}{ Characteristic species of Association } \\
\hline Astragalus strictifolius & 11 & +2 & +2 & 11 & 11 & 12 & 22 & 22 & 22 & +2 & V \\
\hline Teucrium polium & +2 & 22 & 22 & 22 & 11 & . & . & 11 & +2 & +2 & VI \\
\hline Asphodelus aestivus & 12 & 33 & 33 & & & +2 & +2 & & & & III \\
\hline \multicolumn{12}{|c|}{ Characteristic species of Allians Agropyro-Stachyon } \\
\hline Euphorbia denticulata & +1 & . & +1 & . & +1 & . & +2 & +2 & +2 & . & III \\
\hline Centaurea solstitialis subsp. solstitialis & . & +2 & . & . & +2 & +2 & . & . & . & +1 & II \\
\hline Bromus japonicus subsp. japonicus & +1 & +2 & $\cdot$ & $\cdot$ & 33 & +1 & +2 & +1 & . & & III \\
\hline \multicolumn{12}{|c|}{ Characteristic species of Ordo Astragalo-Bromotalia } \\
\hline Astragalus lamarckii & 11 & +2 & +2 & 22 & 22 & 11 & 11 & 11 & +2 & +2 & IV \\
\hline Bromus danthoniae & +2 & +2 & +1 & +1 & . & . & . & +1 & +1 & +2 & III \\
\hline Scutellaria tomentosa & +1 & +1 & $\cdot$ & $\cdot$ & $\cdot$ & $\cdot$ & +2 & +1 & +1 & +1 & II \\
\hline \multicolumn{12}{|c|}{ Characteristic species of Classis Astragalo-Bromotea } \\
\hline Arrhenatherum kotschyi & +2 & +2 & . & . & +2 & +2 & . & . & +1 & 11 & II \\
\hline Phlomis kurdica & +2 & . & . & +2 & +1 & +1 & . & +1 & . & . & II \\
\hline Gypsophila antari & +1 & . & +1 & . & . & . & +1 & . & . & . & I \\
\hline Stipa holosericea & . & . & . & +1 & +2 & +2 & +2 & +2 & . & . & II \\
\hline \multicolumn{12}{|c|}{ Participants } \\
\hline Poa bulbosa & +1 & +1 & +2 & +2 & + & +1 & +2 & +2 & +2 & +2 & II \\
\hline Trifolium speciosum & . & . & +2 & . & +2 & +2 & +1 & . & . & +2 & II \\
\hline Torilis arvensis & +1 & +1 & +1 & . & . & . & . & +1 & +2 & +1 & II \\
\hline Anthemis haussknechtii & +1 & +1 & +1 & $\cdot$ & & $\cdot$ & +1 & . & . & . & I \\
\hline Lolium rigidum & . & . & . & +1 & +1 & +2 & +2 & . & +2 & . & I \\
\hline Capparis spinosa & . & . & . & . & . & +1 & +1 & . & . & . & I \\
\hline Convolvulus arvensis & . & . & . & . & +1 & +2 & . & . & . & . & I \\
\hline
\end{tabular}

The association contain one layer composed of grass and average length was between 20 and $30 \mathrm{~cm}$. The plant cover was changing between 40 and $60 \%$.

Classis: Astragalo-Bromotea Quezel 1973

Ordo: Astragalo-Bromotalia Quezel 1973

Alliance: Agropyro-Stachyon Quezel 1973

This association was spreading out on the calcereous main rock, and gravelled and small pit areas. According to chemical and physical properties of soils, $\mathrm{pH}$ was: 7.28 7.71 , the rate of lime $\left(\mathrm{CaCO}_{3}\right)$ was $3.8 \%$, phosphorus $\left(\mathrm{P}_{2} \mathrm{O}_{5}\right)$ was $5.2 \mathrm{~kg} / \mathrm{dec}$.; potassium $\left(\mathrm{K}_{2} \mathrm{O}\right)$ was $118.8 \mathrm{~kg} / \mathrm{dec}$. and organic component was $2.48 \%$. (Table 3). The yield of pasture was low, between 0.5 and $1.0 \mathrm{ha}^{-1}$, because of excessive grazing. 20 taxa were determined.

\section{Cynodo dactyloni-Brometum danthoniae ass. nova}

The characteristic species of the association were Cynodon dactylon and Bromus danthoni. It was defined in stream beds and depressed places on the southwest of İkizce village at Fatik mountains. The gradient was low (5-10\%), and the altitude was between 
600 and 700 m. 10 sample parcels were used. This association composed of only grass layer and the average length was between 10 and $20 \mathrm{~cm}$., covering was $60-65 \%$ and taxon number was poor (between 7 and 13) (Table 5).

Classis: Astragalo-Bromotea Quezel 1973

Ordo: Astragalo-Bromotalia Quezel 1973

Alliance: Agropyro-Stachyon Quezel 1973

This association was spread out at red-brown soils. According to chemical and physical properties of soils; $\mathrm{pH}$ was: 7.42 , the rate of lime $\left(\mathrm{CaCO}_{3}\right)$ was $6.8 \%$, phosphorus $\left(\mathrm{P}_{2} \mathrm{O}_{5}\right)$ was $4.5 \mathrm{~kg} \mathrm{dec}^{-1}$; potassium $\left(\mathrm{K}_{2} \mathrm{O}\right)$ was $108.0 \mathrm{~kg} \mathrm{dec}^{-1}$. and organic component was found as $2.35 \%$ (Table 3). The yield of pasture was $1.0 \mathrm{ha}^{-1}$

The association Cynodo datyloni-Brometum danthoniae is composed of 27 taxa Table 5).

Table 5. Cynodo dactyloni-Bromoetum danthoniae ass. nova. Thypus: Sample Parcel 64

\begin{tabular}{|c|c|c|c|c|c|c|c|c|c|c|c|}
\hline Sample parcel No & $* *$ & 63 & 64 & 69 & 71 & 74 & 75 & 76 & 78 & 80 & \\
\hline Square size $\left(\mathrm{m}^{2}\right)$ & 50 & 50 & 50 & 50 & 50 & 50 & 50 & 50 & 50 & 50 & \\
\hline Altitude (m) & 470 & 485 & 480 & 485 & 490 & 485 & 480 & 458 & 500 & 505 & \\
\hline Gradient $(\%)$ & 10 & 20 & 25 & 15 & 20 & 25 & 15 & 20 & 25 & 20 & 巳 \\
\hline Direction & $\mathrm{NE}$ & $\mathrm{NE}$ & $\mathrm{NE}$ & NE & NE & $\mathrm{N}$ & $\mathrm{N}$ & $\mathrm{N}$ & NE & NE & $\vec{\Xi}$ \\
\hline Length of cover $(\mathrm{cm})$ & 15 & 15 & 10 & 10 & 15 & 15 & 10 & 15 & 10 & 20 & ⿷匚 \\
\hline Number of species & 13 & 13 & 13 & 12 & 13 & 8 & 12 & 12 & 7 & 10 & \\
\hline Cover $(\%)$ & 65 & 60 & 65 & 65 & 60 & 60 & 65 & 55 & 55 & 65 & \\
\hline \multicolumn{12}{|c|}{ Characteristic species of Association } \\
\hline Cynodon dactylon & . & +2 & 22 & 33 & 33 & 22 & 23 & 23 & +2 & +2 & $\mathrm{~V}$ \\
\hline Bromus danthoniae & +1 & +2 & +1 & +2 & +2 & 11 & 11 & 22 & 11 & 12 & IV \\
\hline Bromus japonicus & +2 & +1 & +2 & 11 & 11 & 11 & +2 & . & . & . & III \\
\hline \multicolumn{12}{|c|}{ Characteristic species of Allians Agropyro-Stachyon } \\
\hline Avena eriantha & +1 & +1 & +1 & +2 & +2 & +2 & +2 & . & . & . & III \\
\hline Eryngium creticum & +1 & . & +1 & . & +1 & . & . & +1 & +1 & +1 & II \\
\hline Cichorium glandulosum & . & . & . & +1 & . & . & $\cdot$ & +1 & . & +1 & $\mathrm{I}$ \\
\hline \multicolumn{12}{|c|}{ Characteristic species of Ordo Astragalo-Bromotalia } \\
\hline Astragalus lamarckii & . & . & +2 & +1 & +1 & +2 & +2 & +2 & +2 & +2 & III \\
\hline Bromus rubens & +2 & +2 & +2 & . & . & . & +1 & +1 & . & . & II \\
\hline Euphorbia characias subsp. wullfenii & . & . & . & +1 & +1 & +1 & . & . & . & . & $\mathrm{I}$ \\
\hline \multicolumn{12}{|c|}{ Characteristic species of Classis Astragalo-Bromotea } \\
\hline Aegilops cylindirica & +2 & +2 & & $\cdot$ & . & . & . & +1 & +2 & $\cdot$ & II \\
\hline Hordeum bulbosum & $\cdot$ & & +1 & +2 & +2 & . & . & . & . & +2 & I \\
\hline Ziziphora capitata & +1 & +1 & +1 & +2 & . & . & . & . & . & . & I \\
\hline Scrophularia canina subsp. bicolor & +1 & +1 & . & . & . & . & +1 & . & . & . & I \\
\hline \multicolumn{12}{|c|}{ Participants } \\
\hline Scandix pecten-veneris & . & . & . & +1 & +1 & +1 & +2 & +2 & +2 & +2 & III \\
\hline Verbascum kotschyi & . & +1 & +1 & . & . & . & $\cdot$ & . & . & +1 & $\mathrm{I}$ \\
\hline Centaurea consanguinea & . & & +1 & . & . & . & +1 & +1 & +1 & . & I \\
\hline Veronica beccabunga subsp. beccabunga & +1 & +1 & & . & +1 & . & . & . & . & . & I \\
\hline Ranunculus arvensis & 11 & +1 & +2 & +2 & +2 & +1 & . & . & . & . & II \\
\hline Secale sylvestris & . & . & . & . & . & . & +1 & +1 & +1 & . & I \\
\hline
\end{tabular}


Allium flavum subsp. tauricum var tauricum

Senecio vulgaris

Crepis reuterana subsp. reuterana

Trifolium tomentosum

Hypecoum pendulum

Avena sterilis subsp. sterilis

Hypericum capitatum var. capitatum

\section{Phlomido bruguieri-Thymbretum spicatae ass. nova}

Phlomis bruguieri and Thymbra spicata subsp. Spicata were the characteristic species of this association. It was spread out on the south part of the Fatik mountain, between Ikizce village and Altun mezra village. The gradient was 15-30\% and the altitude was between 610 and $850 \mathrm{~m}$ (Table O).

The association contained only one layer like the others, grass layer. The average length was $12-25 \mathrm{~cm}$ and rate of covering was $50-60 \%$ (Table 6).

Classis: Astragalo-Bromotea Quezel 1973 1984

Ordo: Onobrychido armenae-Thymetalia leucostomi Akman, Ketenoğlu Quezel

Alliance: Phlomido armeniaceae-Astragalion microcephali Akman, Ketenoğlu Quezel 1986

It was spreading out at red-brown soils. According to chemical and physical properties of soils, $\mathrm{pH}$ was: 7.70 , the rate of lime $\left(\mathrm{CaCO}_{3}\right)$ was $3.8 \%$, phosphorus $\left(\mathrm{P}_{2} \mathrm{O}_{5}\right)$ was $5.3 \mathrm{~kg} \mathrm{dec}^{-1}$; potassium $\left(\mathrm{K}_{2} \mathrm{O}\right)$ was $118.8 \mathrm{~kg} \mathrm{dec}^{-1}$ and organic component was found as $2.48 \%$ (Table 3). The yield of pasture was 1.0-1.5 ha 29 taxa were determined from this association (Table 6 ).

Table 6. Phlomido bruguieri-Thymbretum spicatae ass. nova Thypus: Sample Parcel 17

\begin{tabular}{|c|c|c|c|c|c|c|c|c|c|c|c|c|c|c|}
\hline Sample parcel No & 10 & 13 & 14 & 15 & 17 & 19 & 21 & 22 & 28 & 30 & 33 & 65 & 66 & \\
\hline Square size $\left(\mathrm{m}^{2}\right)$ & 50 & 50 & 50 & 50 & 50 & 50 & 50 & 50 & 50 & 50 & 50 & 50 & 50 & \\
\hline Altitude (m) & 610 & 605 & 707 & 710 & 715 & 720 & 725 & 810 & 815 & 750 & 820 & 822 & 815 & \\
\hline Gradient $(\%)$ & 20 & 15 & 25 & 20 & 25 & 35 & 30 & 20 & 20 & 25 & 30 & 30 & 30 & 过 \\
\hline Direction & SE & S & S & $\mathrm{S}$ & $\mathrm{S}$ & SE & SE & $\mathrm{SE}$ & $\mathrm{SE}$ & SE & SE & SE & $\mathrm{S}$ & ఫ్ర \\
\hline Length of cover $(\mathrm{cm})$ & 25 & 20 & 20 & 25 & 20 & 20 & 25 & 20 & 25 & 20 & 20 & 20 & 25 & \\
\hline Number of species & 12 & 12 & 10 & 11 & 15 & 9 & 9 & 9 & 12 & 7 & 9 & 9 & 7 & \\
\hline Cover $(\%)$ & 50 & 55 & 55 & 60 & 60 & 60 & 65 & 55 & 55 & 50 & 50 & 60 & 55 & \\
\hline \multicolumn{15}{|c|}{ Characteristic species of Association } \\
\hline Phlomis bruguieri & +2 & +2 & 22 & 22 & 11 & 11 & 22 & 22 & 11 & +2 & +2 & +2 & +1 & $\mathrm{~V}$ \\
\hline Thymbra spicata & +2 & +2 & +2 & +1 & +1 & 11 & 11 & 11 & +2 & +2 & +1 & +1 & +2 & IV \\
\hline \multicolumn{15}{|c|}{ Characteristic species of Allians Phlomido armeniaceae-Astragalion microcephali } \\
\hline Hordeum murinum subsp. glaucum & +2 & +2 & 22 & 22 & 11 & 11 & 11 & +1 & +2 & +2 & 22 & 11 & 11 & IV \\
\hline Phleum phleoides & +1 & +2 & +2 & +2 & +2 & . & . & . & • & . & +2 & . & . & III \\
\hline Teucrium parviflorum & +2 & & & . & +2 & . & $\cdot$ & . & +2 & . & . & . & . & II \\
\hline Euphorbia characias subsp. wullfenii & . & & & . & & . & +2 & +2 & & & +2 & . & . & I \\
\hline
\end{tabular}




\begin{tabular}{|c|c|c|c|c|c|c|c|c|c|c|c|c|c|c|}
\hline \multicolumn{15}{|c|}{ Characteristic species of Ordo Onobrychido armenae-Thymetalia leucostomi } \\
\hline Onobrychis caput-galli & +2 & +2 & +1 & +2 & +2 & +2 & +2 & +2 & +2 & . & . & +2 & +2 & IV \\
\hline Bromus scoparius & . & +2 & +2 & +2 & +1 & +1 & +1 & +1 & +1 & +2 & +2 & +1 & . & III \\
\hline Medicago rigidula & +1 & +1 & $\cdot$ & & . & . & . & . & +1 & +1 & . & . & . & I \\
\hline \multicolumn{15}{|c|}{ Characteristic species of Classis Astragalo-Bromotea } \\
\hline Bromus japonicus & . & +2 & +2 & +2 & +1 & +1 & +1 & +2 & +2 & +2 & +2 & +2 & +2 & III \\
\hline Satureja hortensis & +1 & +1 & +1 & +2 & +2 & +2 & . & . & . & . & . & . & +2 & II \\
\hline Scabiosa calocephala & +1 & +1 & +1 & +2 & +2 & & . & . & . & . & +2 & . & . & II \\
\hline \multicolumn{15}{|c|}{ Participants } \\
\hline Hypecoum imberbe & . &. & . & . & +1 & . & . & . & . & . & . & . & . & I \\
\hline Torilis tenella & . & . & . & . & $\cdot$ & . & +1 & . & . & . & . & . & . & I \\
\hline $\begin{array}{c}\text { Xanthium strumarium subsp. } \\
\text { strumarium }\end{array}$ & . & . & $\cdot$ & . & $\cdot$ & $\cdot$ & $\cdot$ & +1 & . & . & . & +1 & . & I \\
\hline Cynosurus echinatus & +1 & . & . & . & . & +1 & . & . & . & . & . & . & . & I \\
\hline $\begin{array}{c}\text { Eremopyrum bonaepartis subsp. } \\
\text { bonaepartis }\end{array}$ & . & +1 & . & . & +1 & . & . & . & . & . & . & . & . & I \\
\hline Urginea maritima & . & . & . & . & $\cdot$ & . & . & . & . & & +1 & . & . & I \\
\hline Secale sylvestris & . & . & . & . & . & . & . & . & . & . & . & +1 & . & I \\
\hline Gagea luteoides & +2 & . & · & . & $\cdot$ & . & . & . & . & . & . & . & . & I \\
\hline Trfolium stellatum & . & . & . & . & +1 & . & . & . & . & . & . & . & . & I \\
\hline Crepis reuterana subsp. reuterana & . & . & $\cdot$ & . & . & . & +1 & . & . & . & . & . & $\cdot$ & I \\
\hline Sedum caespitosum & . & . & +1 & . & . & . & . & . & $\cdot$ & . & . & . & +1 & I \\
\hline Echinops microcephalus & . & . & . & . & +1 & . & . & +1 & +1 & . & . & . & . & I \\
\hline Eryngium creticum & +1 & . & . & +1 & · & $\cdot$ & . & . & +1 & $\cdot$ & & . & . & I \\
\hline Capsella bursa-pastoris & . & . & . & & . & +1 & . & . & $\cdot$ & +1 & . & . & . & I \\
\hline Thlaspi perfoliatum & +1 & +1 & . & +1 & . & . & . & . & . & . & . & . & . & I \\
\hline Velezia rigida & . & . & . & . & . & . & . & . & . & . & +1 & . & . & I \\
\hline
\end{tabular}

\section{Prosopo farctae-Avenetum erianthae ass. nova}

The characteristic species were Prosopis farcta, Avena eriantha and Bromus scoparius. It was determined from the Northwest of Küçük Güneş, Aşağ Güneş and Yukarı Güneş villages of Fatik Mountain at 450-500 m altitudes.

The association composed of only grass layer except Prosopis farcta, the average length was between 15 and $30 \mathrm{~cm}$ and the covering was between 50 and $65 \%$. The number of taxon was between 12 and 18 (Table 7).

Classis: Astragalo-Bromotea Quezel 1973

Ordo: Astragalo-Bromotalia Quezel 1973

Alliance: Prosopo-Bromuon ass. nova

This association was spreading out at red-brown soils. According to chemical and physical properties of soils, $\mathrm{pH}$ was: 7.28 , the rate of lime $\left(\mathrm{CaCO}_{3}\right)$ was $2.6 \%$, phosphorus $\left(\mathrm{P}_{2} \mathrm{O}_{5}\right)$ was $4.9 \mathrm{~kg} \mathrm{dec}^{-1}$; potassium $\left(\mathrm{K}_{2} \mathrm{O}\right)$ was $179.2 \mathrm{~kg} \mathrm{dec}^{-1}$ and organic component was measured as $1.44 \%$ (Table 3). The yield of pasture was $1.0-1.5 \mathrm{ha}^{-1} 26$ taxa were determined (Table 7). 
Table 7. Prosopio farctae-Avenetum erianthae ass. nova Thypus: Sample Parcel 9

\begin{tabular}{|c|c|c|c|c|c|c|c|c|c|c|c|c|c|c|}
\hline Sample parcel No & 2 & 5 & 6 & 8 & 9 & 11 & 12 & 16 & 18 & 20 & 25 & 26 & 27 & \\
\hline Square size $\left(\mathrm{m}^{2}\right)$ & 50 & 50 & 50 & 50 & 50 & 50 & 50 & 50 & 50 & 50 & 50 & 50 & 50 & \\
\hline Altitude (m) & 450 & 455 & 460 & 470 & 475 & 480 & 485 & 490 & 495 & 500 & 505 & 510 & 515 & \\
\hline Gradient (\%) & 10 & 15 & 10 & 10 & 10 & 10 & 15 & 15 & 15 & 10 & 10 & 10 & 15 & (1) \\
\hline Direction & $\mathrm{E}$ & $\mathrm{E}$ & $\mathrm{E}$ & SE & SE & SE & SE & SE & SE & SE & SE & SE & SE & \\
\hline Length of cover $(\mathrm{cm})$ & 25 & 20 & 20 & 25 & 30 & 15 & 15 & 25 & 15 & 15 & 25 & 25 & 20 & \\
\hline Number of species & 18 & 13 & 12 & 13 & 13 & 12 & 12 & 13 & 13 & 12 & 13 & 13 & 14 & \\
\hline Cover $(\%)$ & 55 & 50 & 55 & 50 & 50 & 45 & 50 & 60 & 55 & 50 & 45 & 50 & 50 & \\
\hline \multicolumn{15}{|c|}{ Characteristic species of Association } \\
\hline Prosopis farcta & +2 & +2 & 33 & 33 & 22 & 22 & 11 & 11 & 11 & 22 & 22 & 33 & 33 & $\mathrm{~V}$ \\
\hline Bromus scoparius & +1 & +1 & +2 & . & . & 22 & 22 & +2 & +2 & +1 & +1 & +2 & 22 & IV \\
\hline Avena eriantha & +2 & +2 & 22 & 22 & 22 & +2 & +2 & +2 & +2 & +2 & +2 & & +1 & IV \\
\hline \multicolumn{15}{|c|}{ Characteristic species of Allians Prosopo-Bromиon*** } \\
\hline Phlomis bruguieri & +2 & +2 & +2 & +2 & +2 & +1 & +1 & +2 & +2 & +2 & +2 & +2 & +2 & III \\
\hline $\begin{array}{r}\text { Hordeum } n \\
\text { subsp. glc }\end{array}$ & +1 & +1 & +2 & +2 & 11 & 11 & 11 & 11 & 11 & +1 & +1 & & & III \\
\hline Salvia sclarea & +1 & & & & +1 & +1 & +1 & +2 & +2 & +2 & +2 & & & II \\
\hline \multicolumn{15}{|c|}{ Characteristic species of Ordo Astragalo-Bromotalia } \\
\hline Astragalus lamarckii & +1 & +2 & +2 & +2 & +2 & +2 & +2 & +2 & . & & +2 & & & II \\
\hline s japonicus & +1 & +1 & 11 & +2 & +1 & 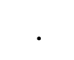 & . & . & +1 & +2 & . & +1 & 12 & II \\
\hline iculatum & +1 & +1 & +1 & 22 & 22 & 22 & 22 & & & & 11 & 11 & & III \\
\hline \multicolumn{15}{|c|}{ Characteristic species of Classis Astragalo-Bromotea } \\
\hline Phleum phleoides & +2 & +2 & +2 & +2 & +1 & +1 & +1 & +2 & +2 & +1 & +1 & +1 & +1 & II \\
\hline Phlon & +2 & +2 & & . & +2 & +2 & & . & +2 & +2 & +2 & & . & II \\
\hline Cichorium glandulosum & +1 & & +1 & +1 & & & & +1 & +1 & +1 & & & . & $\mathrm{I}$ \\
\hline \multicolumn{15}{|c|}{ Participants } \\
\hline Althea officinalis & +1 & +1 & +1 & +1 & +1 & 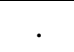 & +2 & +2 & +2 & . & . & . & . & II \\
\hline Verbascum kotschyi & & & & +1 & +2 & +2 & +1 & +1 & +1 & . & . & +1 & +1 & II \\
\hline $\begin{array}{l}\text { Linum pubescens subsp. } \\
\text { pubescens }\end{array}$ & +1 & . & . & +1 & . & & . & . & . & . & +1 & & . & I \\
\hline $\begin{array}{c}\text { Euphorbia szovitsii var. } \\
\text { szovitsii }\end{array}$ & & & & $\cdot$ & . & & . & . & +1 & +1 & +1 & & . & I \\
\hline Eryngium campestre & & +1 & +1 & . & . & . & . & . & . & . & . & . & +1 & I \\
\hline $\begin{array}{l}\text { Hypericum capitatum } \\
\text { var. capitatum }\end{array}$ & +1 & $\cdot$ & & . & . & & • & +1 & . & . & . & +1 & & I \\
\hline Trifolium tomentosum & & . & . & . & +1 & & . & . & . & . & . & +1 & . & I \\
\hline Hordeum bulbosum & & . & & & & & +1 & . & . & . & . & & +1 & I \\
\hline Aegilops cylindirica & & . & . & +1 & . & +1 & . & . & . & . & . & +1 & . & I \\
\hline Torilis arvensis & & & & & & & & . & . & +1 & & & . & I \\
\hline $\begin{array}{l}\text { Carduus pycnocephalus } \\
\text { subsp. albidus }\end{array}$ & +1 & & . & . & . & & . & . & . & . & +1 & & +1 & I \\
\hline Anthemis haussknechtii & & +1 & & . & . & & . & . & . & . & & +1 & +1 & I \\
\hline $\begin{array}{l}\text { Erodium cicutarium } \\
\text { subsp. cicutarium }\end{array}$ & +1 & & & . & . & & & . & . & . & & +1 & +1 & I \\
\hline Lappula barbata & & & +1 & & . & & & +1 & . & & & +1 & +1 & I \\
\hline Secale sylvestre & & & & +1 & & & & & . & . & & & +1 & I \\
\hline
\end{tabular}




\section{Astragalo strictifolii-Salvietum sclarea ass. nova}

Astragalus strictifolius var. strictifolius and Salvia sclarea were the characteristic taxa of this association. It was determined between Fatik and Ikizce villages at northeast direction. The altitude was $600-750 \mathrm{~m}$ and the gradient was between 20 and $35 \%$. The association composed of only grass layer except Astragalus strictifolius var. strictifolius. The average length was between 20 and $25 \mathrm{~cm}$ and the covering was between 50 and 65\%. The number of taxon was between 10 and 18 (Table 8).

Classis: Astragalo-Bromote Quezel 1973

Ordo: Astragalo-Bromotalia Quezel 1973

Alliance: Agropyro-Stachyon Quezel 1973

This association was spreading out at the red-brown soils on the arid stone. According to chemical and physical properties of soils, $\mathrm{pH}$ was: 7.42 , the rate of lime $\left(\mathrm{CaCO}_{3}\right)$ was $6.8 \%$, phosphorus $\left(\mathrm{P}_{2} \mathrm{O}_{5}\right)$ was $4.5 \mathrm{~kg} \mathrm{dec}^{-1}$; potassium $\left(\mathrm{K}_{2} \mathrm{O}\right)$ was $108.0 \mathrm{~kg}$ $\mathrm{dec}^{-1}$ and organic component was measured as $2.35 \%$ (Table 3). The yield of dry grass was between 300 and $400 \mathrm{~g} \mathrm{~m}^{-2} 35$ taxa were determined (Table 8).

Table 8. Astragalo strictifolii-Salvietum sclarea ass. nova Thypus: Sample Parcel 36

\begin{tabular}{|c|c|c|c|c|c|c|c|c|c|c|c|c|c|c|}
\hline Sample parcel No & 1 & 3 & 4 & 36 & 38 & 44 & 45 & 47 & 49 & 51 & 52 & 54 & 58 & \\
\hline Altitude (m) & 50 & 50 & 50 & 50 & 50 & 50 & 50 & 50 & 50 & 50 & 50 & 50 & 50 & \\
\hline Gradient (\%) & 510 & 520 & 530 & 540 & 550 & 555 & 557 & 560 & 565 & 567 & 570 & 571 & 572 & \\
\hline Direction & 30 & 35 & 20 & 20 & 25 & 30 & 30 & 35 & 35 & 30 & 35 & 30 & 25 & I \\
\hline Length of cover $(\mathrm{cm})$ & SE & SE & SE & $\mathrm{S}$ & S & $\mathrm{S}$ & SW & SW & SW & $\mathrm{S}$ & $\mathrm{S}$ & $\mathrm{S}$ & $S$ & $\vec{d}$ \\
\hline Number of species & 25 & 20 & 20 & 25 & 30 & 35 & 35 & 30 & 30 & 25 & 30 & 25 & 25 & \\
\hline Cover $(\%)$ & 13 & 12 & 15 & 16 & 16 & 13 & 13 & 9 & 12 & 10 & 15 & 12 & 12 & \\
\hline Altitude (m) & 50 & 55 & 55 & 60 & 65 & 60 & 60 & 55 & 55 & 60 & 60 & 60 & 50 & \\
\hline \multicolumn{15}{|c|}{ Characteristic species of Association } \\
\hline $\begin{array}{l}\text { Astragalus strictifolius var. } \\
\text { strictifolius }\end{array}$ & 12 & 12 & 22 & +1 & +1 & 22 & +1 & +2 & 22 & 22 & 22 & 11 & 11 & IV \\
\hline Salvia sclarea & 12 & . & 12 & 11 & +1 & +1 & +2 & +2 & 11 & 22 & +2 & +2 & 11 & IV \\
\hline \multicolumn{15}{|c|}{ Characteristic species of Allians Agropyro Stachyon } \\
\hline Thymbra spicata & . & . & +2 & 22 & 33 & +2 & +2 & +2 & +2 & 22 & 22 & 11 & 11 & IV \\
\hline Phlomis kurdica & +2 & +2 & +2 & +2 & +2 & & . & . & $\cdot$ & . & +2 & +2 & & III \\
\hline $\begin{array}{l}\text { Euphorbia characias subsp. } \\
\text { wullfenii }\end{array}$ & . & . & +2 & +2 & +2 & +2 & +2 & . & . & . & & . & +2 & II \\
\hline Stipa holosericea & . & . & . & & . & & & . & +2 & +2 & +2 & . & & II \\
\hline \multicolumn{15}{|c|}{ Characteristic species of Ordo Astragalo-Bromotalia } \\
\hline Festuca callieri subsp. callieri & +2 & +2 & +2 & +2 & +2 & +2 & $\cdot$ & . & . & . & . & . & . & II \\
\hline Astragalus lamarckii & 22 & +2 & +2 & 22 & 22 & . & . & . & 22 & 11 & 11 & 22 & 11 & IV \\
\hline Phlomis ssp. & +2 & +2 & +2 & . & . & . & +2 & +2 & +2 & . & +2 & . & & III \\
\hline \multicolumn{15}{|c|}{ Characteristic species of Classis Astragalo-Bromotea } \\
\hline Eryngium creticum & & e & +2 & +2 & +2 & +2 & +2 & & . & +2 & +2 & . & +2 & III \\
\hline Teucrium polium & +2 & +2 & . & $\cdot$ & +2 & +2 & +2 & +2 & . & . & $\cdot$ & $\cdot$ & . & II \\
\hline Trifolium speciosum & & $\cdot$ & $\cdot$ & +2 & +2 & +2 & . & . & . & $\cdot$ & +2 & +2 & +2 & II \\
\hline Ranunculus arvensis & +2 & . & +2 & . & . & . & +1 & +1 & +1 & +1 & . & . & & $\mathrm{I}$ \\
\hline
\end{tabular}




\begin{tabular}{|c|c|c|c|c|c|c|c|c|c|c|c|c|c|c|}
\hline \multicolumn{15}{|c|}{ Participants } \\
\hline Eminium rauwolffii var. rauwolffii & +2 & +2 & +2 & +2 & +2 & +2 & +2 & +1 & +1 & +1 & . & . & +2 & III \\
\hline Muscari comosum & +2 & +2 & +2 & . & . & . & . & . & . & +1 & +1 & +1 & . & II \\
\hline Gagea luteoides & +2 & +2 & +2 & +1 & +2 & +2 & +2 & . & . & & & +1 & +1 & III \\
\hline $\begin{array}{l}\text { Erodium cicutarium subsp. } \\
\text { cicutarium }\end{array}$ & $\cdot$ & 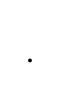 & & . & +1 & +2 & . & . & +1 & +1 & +1 & . & . & I \\
\hline Achillea vermicularis & . & . & - & . & . & $\cdot$ & . & +2 & . & . & $\cdot$ & . & . & I \\
\hline Eryngium campestre & . & $\cdot$ & . & +1 & +2 & +2 & +1 & . & . & . & $\cdot$ & . & . & I \\
\hline Capsella bursa-pastoris & $\cdot$ & & $\cdot$ & $\cdot$ & ${ }^{\circ}$ & & . & . & . & . & +1 & +1 & +1 & I \\
\hline Avena sterilis subsp. sterilis & +2 & +1 & +1 & +2 & +1 & . & . & . & +2 & . & . & . & . & I \\
\hline Echinaria capitata & +1 & +1 & $\cdot$ & $\cdot$ & . & . & . & . & . & . & . & +1 & +1 & I \\
\hline Althea officinalis & . & & . & +2 & . & . & +1 & . & . & . & . & . & +2 & I \\
\hline $\begin{array}{l}\text { Veronica beccabunga subsp. } \\
\text { beccabunga }\end{array}$ & . & +1 & . & . & . & . & . & . & . & . & +1 & +1 & +1 & I \\
\hline Vulpia persica & . & $\cdot$ & . & . & . & +1 & +1 & . & . & . & & . & . & I \\
\hline Bromus japonicus & . & & . & +1 & . & . & . & . & +1 & +1 & +1 & . & . & I \\
\hline $\begin{array}{c}\text { Eremopyrum bonaepartis subsp. } \\
\text { bonaepartis }\end{array}$ & . & . & & . & . & . & +2 & . & . & . & +2 & . & . & I \\
\hline Convolvulus arvensis & . & . & +2 & . & . & . & . & . & . & . & . & . & +1 & I \\
\hline Cichorium glandulosum & . & • & • & $\cdot$ & . & +1 & . & $\cdot$ & . & . & · & . & & I \\
\hline Linum pubescens & . & . & . & +1 & . & . & . & +1 & +1 & . & . & . & & I \\
\hline Aegilops cylindirica & +2 & • & . & $\cdot$ & +2 & . & . & . & . & . & . & . & . & I \\
\hline $\begin{array}{l}\text { Xanthium strumarium subsp. } \\
\text { strumarium }\end{array}$ & . & +1 & & . & . & . & . & +1 & . & . & +1 & . & . & I \\
\hline
\end{tabular}

\section{Conclusion}

In the region, due to excessive and uncontrolled grazing, steppe vegetation has turned into secondary vegetation rather than semi-desert and desert-type vegetation. Therefore, these areas should be controlled and over-grazing should be prevented. In natural pastures, the value of the forage plant and the most suitable pasture capacity should be determined. The productivity of pastures is related to the composition of plant species and habitat factors (Braun-Blanquet, 1964). Morphological structures and ecological habitat forms are one of the most important subjects that should be studied in pasture research projects.

First, the quality and quantity of a pasture should be examined. In the region, plant sociological characteristics of the dominant steppe vegetation change during the seasons. The efemers change the productivity and quality of the pastures. In pastures, forage crops have been reduced due to overgrazing, others spread if inedible to animals. These areas have been abandoned for agriculture and have become barren due to overgrazing. In the future research of these areas, controlled pasture stocking should be carried out together with controlled irrigation for the formation of productive pastures, and even pasture forage crops suitable for barren areas should be selected and trials should be conducted in the area. National and regional pasture management plans should continue to be implemented effectively, sustainable productivity must be ensured and these areas should be put back into service of Turkish livestock. 


\section{REFERENCES}

[1] Adıgüzel, N., Aytaç, Z. (2001): Flora of Ceylanpınar State Farm (Şanlıurfa-Turkey). - Fl Medit 11: 333-361.

[2] Akan, H., Kaya, Ö. F., Eker, I., Cevheri, C. (2005): The Flora of Kaşmer Mountain, Sanliurfa, Turkey. - Turk. J. Bot. 29: 1-20.

[3] Akman, Y. (1990): Climate and Bioclimate. - Palme Publications, Ankara.

[4] Akman, Y., Ketenoglu, O., Geven, F. (2001): Vegetation Ecology and Research Methods. - Ankara University Publications No: 9, Ankara.

[5] Anonymous (1995): Şanlıurfa Province Land Asset. - T. C. General Directorate of Rural Services Directorate Publications, Ankara.

[6] Anonymous (2001): Meteorological Bulletin. - T. C. State Meteorological Service General Directorate No: A-09: 27-29, Ankara.

[7] Aslan, M. (2002): Birecik Dam Lake area plants, hazard classes and conservation. - PhD Thesis, Cukurova University, Institute of Science and Technology, Adana.

[8] Aslan, M. (2015): Succession of steppe areas after fire in the Gap Region of Turkey. Bangladesh J. Bot. 44(4): 489-497.

[9] Aslan, M. (2018): Habitats change in Ceylanpinar State's farm and the dangerous classes of plants (Şanlıurfa-Turkey). - International Journal of Science and Research (IJSR) 7(11): 1766-1772.

[10] Aslan, M., Türkmen, N. (2001): New floristic records for C7 grid square. - Herb Systematic Botanic Journal 8(2): 69-73.

[11] Aslan, M., Türkmen, N. (2003): New floristic records for squares C6 and C7 from Turkey. - Herb Systematic Botanic Journal 10(2): 163-168.

[12] Atamov, V., Cevheri, C., Parmaksız, A. (2004): Şanlıurfa Natural Rangeland Forage Bitkileri. - National Biology Congress XVII: 120, Adana, Turkey.

[13] Atamov, V., Yavuz, M., Cevheri, C., Parmaksız, A., Çen, O., Çetin, E. (2005): "Halophytes and Halophidication In Harran Plain (ŞANLIURFA)". - Biosalina Agriculture \& High Salinity Tolerance, 9-14 January 2005, Muğla, Turkey.

[14] Atamov, V., Aslan, M., Cevheri, C., Cetin, E. (2007): Contribution to the flora of Fatik Mountain. - Asian J. Plant Sci. 6(1): 1-11.

[15] Ayalp, G. (2005): Flora and vegetation of Mezra Town. - Master Thesis, Harran University Institute of Science and Technology, Sanliurfa.

[16] Aydin, N. (2003): Masted Hills flora. - Master Thesis. Harran University Institute of Science and Technology, Şanliurfa.

[17] Aydoğdu, M., Akan, H. (2005): The flora of Kalecik Mountain, Şanlıurfa-Turkey. - Turk. J. Bot. 29: 155-174.

[18] Baytop, A. (2003): History of Botanical Studies in Turkey. - Çetin Printing, İstambul.

[19] Braun-Blanquet, J. (1964): Pflanzensoziologie. Dritte Aufl. - Springer Verlag, Wien.

[20] Çırpıc1, A. (1987): The studies on the flora and vegetation of Turkey. - Turk. J. Bot. 11(2): 217-232.

[21] Çullu, M., Almaca, A., Sahin, Y., Aydemir, S. (2002): Application of GIS monitoring soil salinization in The Harran Plain. - International Conference on Sustainable Land Use and Management, Şanliurfa, Turkey, pp. 326-333.

[22] Davis, P. H. (ed). (1965-1985): Flora of Turkey and the East Aegean Islands. Vol. 1-9. Edinburgh University Press, Edinburgh.

[23] Davis, P. H. (1988): Flora of Turkey and the East Aegean Islands. Vol. 10. - Edinburgh University Press, Edinburgh.

[24] Donner, J. (1990): Distribution Maps to P. H. Davis "Flora of Turkey, 1-10". - Linzer Biol. Beitr. 22(2): 381-515.

[25] Ertekin, S. (2002): Karacadağ Plant Diversity Book. - Sustainable Rural and Urban Development Association Publication, Diyarbakır. 
[26] Ertekin, S., Saya, Ö. (1997): New floristic records for the various grid squares from the Fabaceae. - Tr J of Botany 21: 187-189.

[27] Gençkan, M. (1983): Agriculture of Forage Crops. - Ege University Printing House, Bornova, Izmir.

[28] Güner, A., Özhatay, N., Ekim, T., Baser, K. H. C. (2000): Flora of Turkey and East Aegean Islands. Vol. 11. - Edinburgh University Press, Edinburgh.

[29] Kaya, F. Ö (2002): Flora of Tektek Mountains (Sanliurfa). - Master Thesis, Harran University Institute of Science and Technology, Sanliurfa.

[30] Kaynak, G. (1987): New record for some squares in flora of Turkey. - Nature TU Bot. Magazine 11(2): 118-123.

[31] Kaynak, G. (1989a): Contribution to the flora of Karacadağ (Urfa and Diyarbakır Provinces. - Nature TU Bot. Magazine 13(3): 376-397.

[32] Kaynak, G. (1989b): Ecological and chorological investigations on the ferns of Diyarbakir and surrounding provinces. - Nature TU Bot. Magazine 13(3): 437-451.

[33] Kaynak, G., Ketenoğlu, O. (1980): New fern specimens in South East Anatolian Region. - Ist Univ Science Faculty Mec Series B 45: 199-202.

[34] Malyer, H. (1981): A chorological study on the geophytes of the Iridaceae family of the Diyarbakır region. - Nature Science Journal Basic B 6: 17-20.

[35] Malyer, H. (1983): Chorological and ecological investigations on geophytes of Liliaceae and Iridaceae families in Karacadağ (between Diyarbakır and Urfa). - Nature Science Journal Basic, Series C 7(3): 279-288.

[36] Özhatay, N., Byfield, A., Atay, S. (2003): Turkey's Important Plant Alanları. - WWFTurkey Natural Life Protection Foundation, Istanbul.

[37] Ture, C., Bocuk, H. (2007): An investigation on the diversity, distribution and conservation of Poaceae species growing naturally in Eskisehir province (Central Anatolia-Turkey). - Pak. J. Bot 39(4): 1055-1070.

[38] Türkmen, N., Aslan, M., Düzenli, A. (2002): New records for the various squares in the flora of Turkey. - Journal of Herb Systematic Botany 9(2): 63-68.

[39] Yildırımlı, Ş. (1992): Distribution of new plant to Turkey from various squares. - Nature TU Bot Derg 16: 207-214.

[40] Yildırımlı, Ş. (1994): From Turkey Brassicaceae (Cruciferae) were from New Floris squares for the various family Kayitlar. - T J of Botany 18: 389-392.

[41] Yıldız, B., Aktoklu, E. (1996): New floristic records from C7 square (MalatyaAdiyaman). - Tr J of Botany 20: 207-211.

[42] Zohary, M. (1973): Geobotanical Foundations in the Middle East. Vol. 1-2. - Gustav Fischer Verlag, Stuttgart. 